\title{
A Communication Technique for Swarm- Capable Autonomous Agents
}

\author{
Hans Dermot Doran
}

\begin{abstract}
This paper reports on a communication technique and associated technology designed to achieve embodied and situated communication between swarm-capable autonomous agents. The swarm model chosen was that of a fish school. A RFID based wireless communication system, also capable of measuring both direction of and distance to transmitter, was developed and installed on a Braitenberg vehicle. The communication technique allows vehicles to transmit, at regular intervals, their physiological state to the outside world in general and other vehicles in particular. The information transmitted may be used by other vehicles to decide whether or not to form a school and the communication reach fulfils the prerequisites to enable the vehicle to travel in a school formation.
\end{abstract}

Index Terms- Artificial intelligence, Communication systems, Cooperative systems, Mobile robotics, Robotics, Swarm robotics.

\section{INTRODUCTION}

$\mathrm{S}_{\mathrm{b}}^{\mathrm{N}}$ WARM intelligence and the promise of cooperative behaviour achieving more that the sum of individual effort has inspired much research effort over the last decade or so. The fact that in this decade no satisfactory artificial swarm behaviour has been emulated speaks for the subtle difficulties faced by the researcher. This paper aims to provide a contribution to this theme in the subject area of inter-agent communication and localization.

The paper positions itself in the general theme of the principles of intelligent systems and embodiment as understood by Pfeiffer [1] and within the general terms of situated communication as understood by Støy [2]. The paper begins with the discussion of some concepts, assumptions, opinions and generalities designed to situate a use-case of the work. Some communication concepts are discussed in the next chapter. Details of the technology development are then discussed. The paper proceeds to critically discuss the results before drawing appropriate conclusions and describing further work.

\section{INTRODUCTORY DISCUSSION}

Despite much research on the subject of swarm formation and swarm intelligence, remarkably few, if any, realistically

Manuscript received November 23, 2008.

$\mathrm{H}$ Doran is head of the Real-Time-Ethernet research group at the Institute of Embedded Systems and Professor of Information and Communication technology at the Zürich University of Applied Sciences in Winterthur. (Phone +41 796368133 e-mail: hans.doran@zhaw.ch practical applications have been suggested for swarms of ant or bee-like robots. One of the undoubted advantages of such swarms is that the individual is generally expendable. Unfortunately the relatively high cost of robots means that for practical purposes single, very simple agents are still too expensive to be considered expendable. There are however numerous examples of the utilization of expensively trained intelligent mammals for various tasks, such as dolphins and dogs so a realistic proposition of the first order might appear to be the emulation of animals on this level of the evolutionary scale. The author chooses to imagine a hunting pack of wolves as his "target" scenario.

Wolves have a highly regulated, leadership-based social structure [3]. Furthermore wolves can travel in packs for long distances at substantial speeds before engaging in tactical hunting behaviour. Whilst traveling, wolves are not adverse to physical contact with each other or their surroundings. Faced with an obstacle it is generally up to the individual to take avoidance action; leaning into a neighbour to influence direction, acceleration, deceleration, directional change and jumping over obstacles, including fellow wolves, are all valid judgments an individual may make within the physical confines of its current position in the pack

Robots - and we narrow our scope to wheeled agents, do not generally possess this range of choices and it is far from sure that emulating such capabilities is a cost efficient use of research time so from a robotic point of view we can validly define that pack-like activity takes place in 2-dimensional space. In addition whilst the robots should be robust to minor collisions, engineering efficiency requires collision avoidance so whilst a robot may be designed to complete activities on the tactical level of a wolf, it is clearly desirable to use another model to get the robots to the scene of action.

Flocking behaviour has been studied and simulated - most notably by Reynolds [4] and force-field like collision avoidance algorithms are used in industries, notably in aeronautics, for example [5], where cost is secondary to safety issues but the schooling behaviour of fish poses an attractive model for several reasons not least of which being the large body of research conducted in this area.

School behaviour refers to groups of fish grouped for travel and safety proposes - specifically to confuse predators [6]. The positioning of fish within the school is fluid [7]. The behaviour of fish within a school may be reduced to simple behavioural patterns [8] which may be suited to achievement 
by either emergent behaviour [9] or plain old pre-programmed models. Such models have been well studied, [10], [11] and models based on Aoki's work [12] have been expanded into practically usable ones [13] which avoid the computational effort of Newtonian dynamical equations. Fish exhibit well known acoustical, optical, mechanical and chemical sensitivities and, in terms of fish schools, the task of communication blurs with that of collision avoidance and speed adjustment. Whilst lateral-line sensitivity is not well researched [14] we are able to gain some inspiration by switching species. The clawed frog Xenopus is not able to see in water but is able to detect insects on and in the water using its lateral-line system. It can also localize and identify its prey by the ripples it (insects) makes on the surface of the water; signal processing models have been derived to imitate its capabilities [15]. It has been shown in the same context [16] that stamps produce reproducibly identical signals when dropped into water - a characteristic signal. The assumption that if Xenopus can orientate its prey using the lateral-line system then it can also detect members of its own species appears reasonable.

The current discussion has been centered on sensory systems that primarily uphold school travel. The processes involved for initial school formation, or gathering is also not well considered but, returning to our wolf model; whilst our robot, "loup de terre" so to speak, will move efficiently from A to B using fish schooling dynamics, the joining and possibly peeling-off from the school/pack will probably occur in connection with high level communication - we assert this as being the behavoural interface between wolf and fish. Whilst wolves utter a series of yelps and barks, fish may transmit information optically, mechanically or electrically. Although fish can also detect soluble chemicals, the dispersion rate of such chemicals in water is not fast enough to be of use in fast evasion situations.

We know that fish are sensitive to pressure gradients caused by approaching predators [14] but we may well assume that when in a school these gradients are attenuated or modulated by pressure gradients caused by the swimming action of neighbours. Fish on the outer rims of the school, most exposed to predators will react to the latter's activity and by their physical activity propagate "a message" through the school. We shall assume that the physical activity occurs based on the emotive and physiological states of the fish which triggers activation of an appropriate behavioural pattern and that this behavioural patterns can be recognised as such by their school colleagues.

\section{COMMUNICATION THEORY}

Communication techniques are generally reduced to communication protocols suited for various media, this paper would like to review some basic terms to illustrate the attempt to map imitations of natural communication onto technical communication systems.

We consider a school of fish to consist of a large number of anonymous and homogeneous agents. This implies that directed communication of the form called, in Ethernet, unicast, is not a first order requirement in fish school communications. The communication reach implied by the term broadcast implicitly assumes that all agents receive a communication and may choose whether or not to act on this communication. Broadcast communications in the technical realm are generally considered an inefficient use of bandwidth and processing power as an agent must first determine whether a message is of interest. In asynchronous systems, such as CAN or even Ethernet, bandwidth efficiency - in the face of large number of transmitting nodes, may only be achieved by scheduling designed to avoid collisions [17]. Scheduling implies a master device - something at odds with the notion of a peer-to-peer system as represented by a school. Multicast communication as understood in Ethernet systems allows the reception of frames by a sub-group on the segment. As with unicast and broadcast, media-bandwidth use is not necessarily efficient although processing power is generally conserved. Wireless media generally stipulates more stringent bandwidth conservation and cross-interference requirements than most wired media.

We may therefore model cooperative behaviour depending not only on not knowing the precise identity of a cooperating agent but also on the notion that, due to physical media limitations, an agent cooperates with direct neighbours rather than agents several times removed in geographical location. In considering artificial school communication we might redefine unicast communication to be directed communication with a single, yet possibly anonymous neighbouring agent - the initiator must move to within a physical distance of the intended recipient. Normal state of affairs is interaction, in multicast fashion, to a group of neighbours. This geographical qualification of communication reach has as a consequence that the media must attenuate the signal during its propagation so broadcast communication is an unintentional subset of multicast under the conditions that there is no physical obstacle to signal propagation. Under conditions of attenuating media, broadcast communications are necessarily of low signal strength and may be detected as noise by both friend and foe. [13] also indicates distance values for communication (but not just detection) reach namely attractive-reaction field spanning a radius of 10 times fish body length (BL), the parallelorientation field at $5.0 * \mathrm{BL}$ and the repulsive reaction field at $1.0 *$ BL. Communication reach should therefore be about 10 $* \mathrm{BL}$, detection reach may be much further.

Reducing communication reach to a radius of neighbours conserves both global and local bandwidth. Local bandwidth is dependent on the number of neighbours a fish may have and the rate of change of pressure gradients - in other words speed of swimming. If swimming in a 2-dimensional square formation a single fish may have up to 8 direct neighbours which will each, by physical presence, substantially attenuate or modulate neighbour-generated pressure gradients. Simulations [14] show that the number of expected neighbours 
is in the region of 4-6 depending on the position of the fish in the school. Pressure gradients are caused by tail-fin and associated body movements. Fast fish movement is given at about 3-tail-fin beats a second, any artificial "message" giving a reasonable margin to avoid message collisions in a robotic environment - may not take longer than about $4 \mathrm{~ms}$.

The communication technology described in this paper is designed to achieve these forms of communication reach.

\section{COMMUNICATION TECHNOLOGY}

\section{A. Previous Work}

Previous work is concisely detailed in this section to describe the development path taken and to show different variants of the technology and inspire further work in this direction.

\section{1) Hydron II}

The initial impetuous came from a functional re-design [18] of the Hydron robot [19]. Designed for water operations the spheres communicated with each other using (line-of-sight) LED transmissions. Unfortunately the roll of the robots of in the water meant that line-of-sight communications were severely compromised, communication was disturbed by reflections off the tank walls and a protocol was required to reduce the effects of packet collisions. Finally diffraction makes light based communication underwater impracticable. A short study on communication technologies concluded that an inductive communication system would be more practical as magnetic permeability is virtually the same for both air and water. Accordingly a prototype was built using ASK modulation on a $200 \mathrm{kHz}$ carrier. This managed communication up to 1.8 meters, in good agreement with theoretical values, using a simple communication protocol at baudrate of 605 Baud or maximum 55 messages per second or, more tellingly, each message needed $18.2 \mathrm{~ms}$ "air-time". The (desired) signal degradation by the media is $r^{3}$, inline with magnetic theory.

Localisation, distance and relative position, was achieved by measuring received signal strength using 4 antennae (coils), arranged in a quadrant. A master Hydron II sent a "follow-me" frame and the slave Hydron II would then do precisely that. Whilst the prototype functioned excellently it was felt that several optimizations were required. The first was that since the coils (antennas) had to be wound by hand, significant tuning had to be carried out during commissioning. It was felt that a usable signal should be detectable at around 2.5 meters rather than the current 1.8 meters. An improvement on the angular precision of $\sim 30^{\circ}$ was hoped for and it was hoped the baudrate could be improved upon.

\section{2) RFID Technology}

A second project [20] took these issues to task by upping the carrier frequency to $13.56 \mathrm{MHz}$ common in RFID technology. Thus could the coils be implemented directly on a PCB and the baudrate improved. Experimentation determined that three antennas were sufficient and whilst precision of localization was improved, angles of 10-20 degrees could be determined, the reach was restricted to a distance of approx 70 $\mathrm{cm}$. A look-up table was used to map received signal-strength (from $\mathrm{mV}$ to bits) values to distance (see Fig. 1).

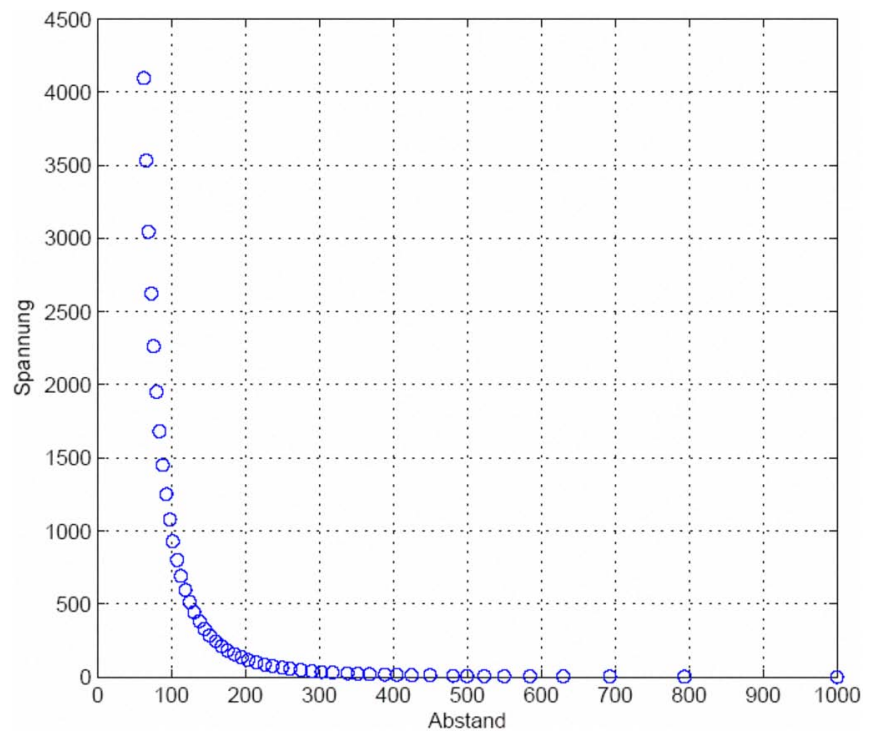

Fig. 1. Signal Strength vs. Distance. Y-axis is signal strength expressed in $\mathrm{mV}$ at $\mathrm{A} / \mathrm{D}$ converter and $\mathrm{X}$-axis is distance from transmitter to receiver in $\mathbf{m m}$.

\section{3) Otto}

Dünner and Kern [21] developed and described the platform "Otto". This, in the context of this paper, is noteworthy for several reasons. Otto is a Type 2 Braitenberg [22] vehicle this vehicle's raison d'etre is given by the direct connection of actuators (drives) and sensors, in this case light sensors. Otto was deliberately constructed using a distributed control system common in the automation industry. Each sensor and each actuator was connected to its own microcontroller which was connected to an Ethernet network. A simplified implementation of the Real-Time-Ethernet (RTE) protocol, Ethernet POWERLINK [23] was used as a communication protocol making each module a Controlled Node $(\mathrm{CN})$. Nodes communicate in cross-traffic mode allowing, for instance, the left hand motor to listen to either the left or right hand light sensor. Cross-traffic requires a bus master to uphold cyclic communication; this is the first known implementation of a POWERLINK bus master as opposed to an application master. This architecture was used to allow later experimentation with motivated behavioral change of Braitenberg vehicles. An observer (in the vocabulary of state machine terminology [24]) captures the light intensity measured by each light sensor, fuzzyfies it and transmits it to other Ottos. In the first version this was achieved using a specially developed POWERLINKover-WLAN protocol - also the first recorded wireless extension of POWERLINK. POWERLINK was used to simplify debugging through guaranteed collision-free time-slot transmission and time qualification of frames. 
The purpose of fuzzyfication, which may be replaced by any other conceivable function, is to make a representation of the physiological state (PS), or indeed emotive state, of one robot available to other robots - perhaps useful in social robotics (for an introductory overview see [25]). This PS is not data as we understand it but information with which, along with other pieces of information, a second vehicle is equipped to make inferences.

Several modifications were subsequently made to the Otto architecture (see Fig. 3). The BusMaster module was replaced with one with an on-board WLAN $^{1}$ port allowing more efficient transfer of debug-data over the WLANPOWERLINK link. A debug/visualization/remote control tool was written [26] to support experimentation. Replacing the WLAN module freed-up a RS232 port to which the torsoLine communication system could be attached.

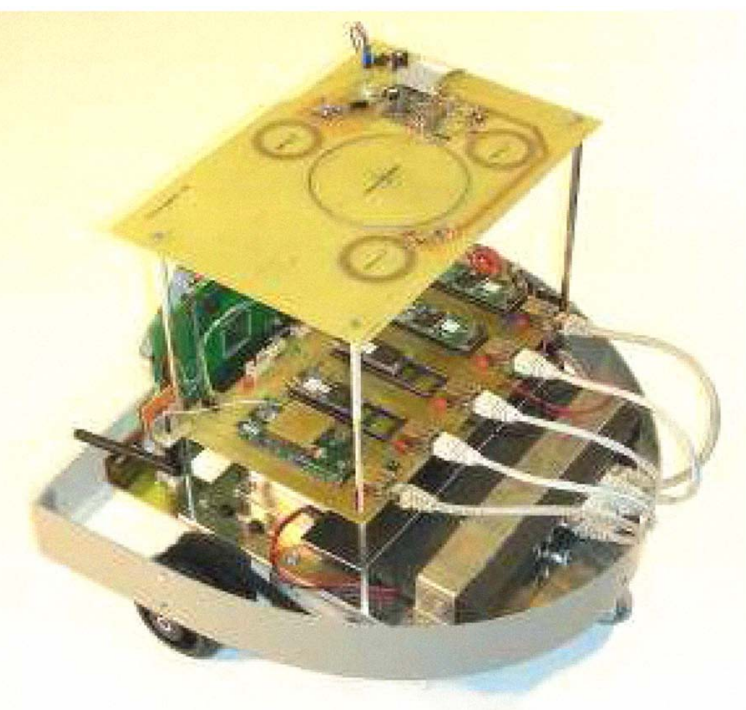

Fig 2. The Modified Otto Platform. torsoLine with the single transmitter and triple receiver antennas is the top PCB. The green vertical PCB is the dsPIC development board. On the third PCB the module on the far left is the WLAN-fitted bus master with the two Coldfire modules to the immediate left. All modules are connected to a hub via Cat5 patch cables.

\section{B. torsoLine}

torsoLine [27] is a redesign of the RFID communication system for the Otto platform. The torsoLine communication controller, a dsPIC is connected to the BusMaster via RS232 link (see Fig. 4). The data-interfaces from torsoLine to BusMaster are the xy coordinates of a detected Otto as well as its PS. In the other direction the PSObserver transmits the PS to the torsoLine at the same rate as the internal POWERLINK cycle (ca. 100ms). The BusMaster acts as POWERLINK managing Node with respect to the internal network and a POWERLINK Controlled Node with respect to the WLAN debug channel.

${ }^{1}$ http://www.dilnetpc.com/dnp0080.htm

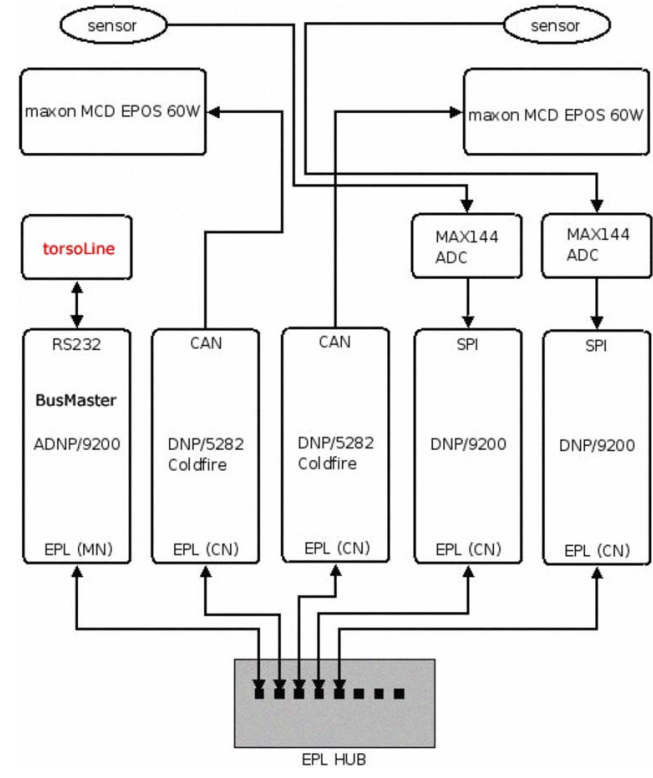

Fig. 3. Modified Control Architecture Otto. The BusMaster was replaced with a WLAN capable ADNP/9200 and the RS232 connection used to connect the torsoLine with the BusMaster

The BusMaster also runs the Vectorisation task which converts received coordinates into a vector, adjusted by the vehicles own trajectory, which indicates in which direction the vehicle ought to travel if it wished to build a swarm. Standard RFID components allow a fairly simple, cost efficient and deterministic, at board level, system to be built. Initial results indicating communication distances of ca. 2.5 meters proved to be optimistic; feedback of magnetic lines in power cables was being measured. Currently communication distance of ca. 1 meter and a baudrate of $106 \mathrm{kBit} / \mathrm{s}$ can be achieved. This can be increased by driving the transmitter with more power and increasing the radius of the transmitter coil. Precision of detection was down to $50 \mathrm{~mm}$ at distances of $800 \mathrm{~mm}$.

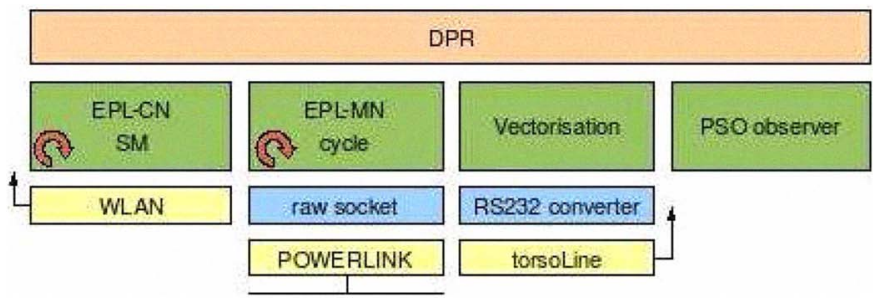

Fig. 4. Software Architecture BusMaster

\section{Swarm Capable Autonomous Agent}

Otto will left to its own devices perform the functionality of a Braitenberg Type $2 b$ vehicle transmitting its physiological state, a measure of its satisfaction with its current illumination, via torsoLine, at a rate determined by the POWERLINK cycle time. The BusMaster may dynamically change the cycle time allowing the PS to be qualified with an excitation rate. An Otto and its current state may be localized with reasonable precision 
within a restricted radius of ca $2 *$ Otto Body Width (see [14]). The radii of the three fields, Attractive-reaction, Parallelorientation and Repulsive-reaction are significantly smaller than the model suggested by [14]. More work is required to determine whether this model is applicable using this technology.

\section{Proof-of-Concept}

For the scope of this paper proof-of-concept is given when under laboratory conditions one Otto can be made to follow a second at the approximate speed of the second. Multiple (behavioral pattern) inputs to the motors can be elegantly handled using an extended Braitenberg architecture [28] but in this case a simple on/off mechanism was used. By using a remote control channel, Otto A was given a given a straight line trajectory designed to take it past Otto B at a distance of ca. $400 \mathrm{~mm}$. Otto B was programmed to drive the two motors with two speed values derived from the movement vector calculated from transmissions from Otto $\mathrm{A}$. So it could be proven that Otto B could understand and react to Otto A, albeit in a trivial manner. Although this merely a repeat of a similar experiment achieved with Hydron II, the importance of the success of this experiment is that a complete communication pathway originating from the simplest physiological state of one agent and directed to the world at large could be detected, localized and understood by agents of the same breed, could be demonstrated.

More work is required to achieve fish school-like travel, speed and direction control.

\section{DISCUSSION OF RESULTS}

\section{A. Embodied and Situated Communication}

The work described in this paper has been framed in terms of embodied and situated communication. Despite the fact that the original aim was to implement a communication system that could function equally well on land and in water, what emerged was a lateral-line-like sensory system allowing the reasonably precise location of a transmitter with a near field region. There are other technologies with these capabilities [29] but without the limitation of communication reach. Communication originates at the physiological level so we may well speak of embodied communication. A purist interpretation of situated communication might insist on air pressure gradients caused by moving vehicles to be measured rather than generating and modulating an artificial magnetic field. Some might think that it would be more appropriate if the magnetic field were "always-on" and indeed the implications of that methodology are worthy of closer attention but the author believes that the achieved is an excellent compromise between engineering-driven and biologically inspired solutions.

\section{B. Pheromone Communication and the "Digital Lamppost"}

What does appear important is that in a particular spectrum of the real-world, namely the magnetic, can be accessed. It is therefore possible for heterogeneous systems to interact with each other. Communication is currently achieved using binary coded PS values. As currently conceived these take the form of a string of 5 16-bit values, currently implemented is a single 16-bit PS value. By no great stretch of the imagination these values may also be modeled as complex pheromones. Using RFID passive tag technology it is possible to construct "digital lampposts" upon which passing agents can "deposit" these pheromones. Enhancing these might allow time-degradation of these pheromones to be achieved allowing agents to deposit warnings or encouragements to each other more in line with natural examples. The author sees this as a more practical alternative to using "real" pheromones [30] and capable of more complex information storage and transfer than [31].

\section{Cross-breed Communication}

Much work has gone into, not all of it successful, attempting to achieve communication between heterogeneous digital systems. Current torso-line communication is achieved by binary coded ASK - by modulating the carrier frequency with an analog signal, think in terms of a heartbeat with its various frequency and amplitude qualified-components, simple filtering technology can be used for partial decoding of transmissions by agents of a different "breed" to the receiver.

\section{Sensing}

Relevant tests have yet to be performed but since the torsoLine is capable of reading its own transmissions it would appear possible that neighbouring animate and inanimate ferromagnetic objects also be detected. Further work and thought must determine whether this would be useful in the particular context of interaction of autonomous agents with either the real-world or with an imaginary world.

\section{E. Far Field Detection}

It may be possible, using different coils, more sensitive amplifiers and tuned filters, to cost-efficiently detect generated magnetic fields in the mid-field - say from near-field to 25 meters. It would be possible for agents to understand that danger was approaching without necessarily being able to identify or localize it with greater precision than a quadrant.

\section{F. Far Field Communication}

Whilst the work described in this paper uses inductive RFID technology for communication within a range of 2.5 meters there appears no reason why for longer range communications, in the range of several tens of meters cannot be achieved by electromagnetic propagation of the same signal using essentially the same back-end electronics but discarding the coils and using $50 \mathrm{Ohm}$ antennae.

\section{G. Engineering Robots}

An aspect worthy of further attention has been touched on in this paper and that is the notion of merging behavioural patterns derived from different animals into one robot. Wolves live in an immediate hierarchical structure with a designated leader, fish prone to schooling activity don't. Wolves don't 
travel in packs for safety but to get from A to B, in contrast many schools of fish exist solely to achieve safety in numbers and have no destination (many do). Yet from an engineering point of view - the engineering of behavioural patterns - it appears efficient to mix behavioural patterns from various animals into one agent. This entails not only architectural, interfacing and methodological aspects but it would appear that knowledge that is not traditionally on the curriculum of engineering schools is also required.

\section{H. Engineering Methodologies}

Usually the engineer decides to use a particular technology based on various requirements and dealing with the disadvantages of that particular technology. The paper anchors the notion of communication reach in a direct relationship with the size of the robot implying that communication reach decreases with decreasing size of robot - a new situation with yet to be considered methodological aspects for most engineers. Additionally the scope for (size) customization of torsoLine has yet to be explored.

\section{CONCLUSION AND FURTHER WORK}

The author and his co-workers, have demonstrated a viable technique and associated technology for embodied and situated communication between autonomous agents.

The next work-package, apart from refinements already discussed is to use this technique and technology in achieving schooling behaviour.

\section{ACKNOWLEDGMENT}

Thanks are due to Prof. Marcel Rupf, Zentrum für Signalverarbeitung und Nachrichtentechnik, Zurich University of Applied Sciences, for help and support on early versions of the work described here. Thanks are also due to SSV Embedded Systems for their sponsorship of several DNP5282 boards.

\section{REFERENCES}

[1] R. Pfeiffer, C. Scheier. Understanding Intelligence. MIT Press, Cambridge, MA. 1999.

[2] K. Støy. "Using Situated Communication in Distributed Autonomous Mobile Robotics" in Proc. $7^{\text {th }}$ Scandinavian Conference on Artificial Intelligence 2001.

[3] L. D. Mech, L. Boitani. Wolves, Behavior, Ecology and Conservation. University of Chicago Press, Chicago, 2003.

[4] C. Reynolds "Flocks Herds and Schools: A Distributed Behavioral Method". Computer Graphics, 21 (4), pp 25-34. July 1987 also http://www.cs.toronto.edu/ dt/siggraph97-course/cwr87// retrieved November 2008.

[5] C. Carbone, U. Ciniglio, F. Corraro, S. Lungo. "A Novel 3D Geometric Algorithm for Aircraft Autonomous Collision Avoidance" in Proc. $45^{\text {th }}$ IEEE Conference on Decision and Control. 2006.

[6] H. Kunz, T. Züblin, C. Hemelrijk. "On prey grouping and predator confusion in artificial fish schools". In Proc. of $10^{\text {th }}$ IInternational Conference on Artificial Life, MIT Press, Cambridge MA, 365-371. 2006

[7] C. Hemelrijk., H. Kunz, "Density distribution and size sorting in fish schools: an individual based model". Behavioral Ecology 2004.

[8] P. Slater. An Introduction to Ethology. Cambridge University Press, Cambridge, 1985.
[9] V. Hafner. H. Kunz, R. Pfeiffer. "An Investigation into Obstacle Avoidance as an Emergent Behaviour from two Different Perspectives". In proc. of the EPSRC/BBSRC International Workshop on BiologicallyInspired Robotics. 2002. Available on http://www.verenahafner.de/papers/emoa.pdf

[10] B. Partidge, T. Pitcher. "The structure and function of fish schools". Scientific American. pp 114-123. June 1982.

[11] M. Zheng, Y, Kashimori, O. Hoshino, K. Fujita. T, Kambara. “ Behaviour pattern (innate action) of individuals in fish schools generating efficient collective evasion from predation". Journal of Theoretical Biology 235 (2005) 153-167.

[12] I. Aoki. "An analysis of the schooling behavior of fish: internal organization and communication processes. In Bulletin Oceanic Research Institute. Tokyo, 1980.

[13] Y. Inada. "Steering mechanism of fish schools". Complexity International 2001. http://www.complexity.org.au/. Retrieved November 2008.

[14] D. Evans, J. Claiborne. The Physiology of Fishes. 3rd Edition. Taylor \& Francis, Boca Raton. 2006.

[15] T. Nolte, M, Sjödin, H, Hansson. "Server-Based Scheduling of the CAN Bus", in Proc. $9^{\text {th }}$ IEEE International Conference on Emerging Technologies and Factory Automation, 2003.

[16] J. Franosch, M. Sobotka, A. Elepfandt, J. von Hemmen. "Minimal Model of Prey Localisation through the Lateral-Line System" in Physical Review Letters. Volume 91, Number 15 October 2003.

[17] B. Claas, H. Münz. "Analysis of surface wave direction by the lateral line system of Xenopus: Source localization before and after inactivation of different parts of the lateral line" in Journal of Computational Physiology. Volume 178. pp 253-268 1996.

[18] M. Bigler, L. Iten. Kommunikationssystem für schwimmende, autonome Roboter. Diploma thesis. Zürich University of Applied Sciences, 2005, unpublished.

[19] E. Ostergaard, D. Christensien, P. Eggenberger, T. Taylor, P. Ottery, H. Lund. "HYDRA: from Cellular Biology to Shape-Changing Artefacts". In Proc. 15th International Conference on Artificial Neural Networks (ICANN 2005), 2005.

[20] S. Gysel, P. Storrer. Distanz und Richtungsschätzung mit RFID. Project report. Zürich University of Applied Sciences 2006, unpublished.

[21] S. Dünner, D. Kern. "Ethernet POWERLINK as a Communication System Internal to and Between Autonomous Angents. In Proc. IEEE International Symposium on Industrial Electronics. 2008.

[22] V. Braitenberg. Vehicles, Experiments in Synthetic Psychology. MIT Press, Cambridge 1984.

[23] Ethernet POWERLINK Communication Profile Specification, EPSG Draft Standard 301, Version 1.1.0. EPSG. Berlin 2008

[24] V. Strejc. State Space Theory of Discrete Linear Control. Academia Praha, Prague, 1981.

[25] F. Michaud, P. Pirjanian, J. Audet, D. Létourneau. "Artificial Emotion and Social Robotics", in (eds.) L. Parker, G. Bekey, J. Barhen, Distributed Autonomous Robotic Systems 4. Springer, Tokyo 2000

[26] T. Levey, A. Stämpfli. Tracking Software. Project Report. Zürich University of Applied Sciences 2008, unpublished.

[27] D. Blattmann, C. Frick. Sensor Fusion. Project report. Zürich University of Applied Sciences 2008, unpublished.

[28] D. Lambrinos, C. Scheier. "Building complete autonomous agents: a case study on categorization" in proc. IEEE/RSJ International Conference on Intelligent Robots and Systems. 1996.

[29] A. De Angelis, M. Dionigi, A. Moschitta, P. Carbone. "A Low-Cost Ultra-Wideband Indoor Ranging Technique" in Proc. IEEE Instrumentation and Measurement Technology Conference. IMTC 2007.

[30] A. Purnamadjaja, R. Russell, "Pheromone Communication: Implementation of Necrophoric Bee Behaviour in a Robot Swarm", Proceedings of the 2004 IEEE Conference on Robotics, Automation and Mechatronics. Singapore.

[31] D. Payton, R. Estkowski, M. Howard. "Pheromone Robotics and the Logic of Virtual Pheromones" in "Swarm Robotics. SAB 2004 International Workshop" LNCS 3342, Springer, New York. 2005. 